\title{
Shen-su-yin, a Traditional Herbal Medicine, Attenuates Lipopolysaccharide-induced Acute Lung Injury in Rats Through Its Anti-inflammatory and Anti-oxidative Effects
}

\section{Peng Xiao \\ Fujian Provincial Hospital \\ Jun Ke \\ Fujian Provincial Hospital \\ Jiuyun Zhang \\ Fujian Provincial Hospital \\ Haijun Zhou \\ Fujian Provincial Hospital}

Wuhong Zheng ( $\nabla$ zhengwuhong@sohu.com )

Fujian Provincial Hospital

\section{Original Research}

Keywords: Shen-su-yin, lipopolysaccharide, acute lung injury, inflammation, oxidative stress

Posted Date: December 6th, 2021

DOI: https://doi.org/10.21203/rs.3.rs-1089681/v1

License: (c) (1) This work is licensed under a Creative Commons Attribution 4.0 International License.

Read Full License 


\section{Abstract}

Most components of Shen-su-yin (SSY), an herbal formula, have anti-inflammatory and antioxidant activities. The present study was designed to investigate potential effects and mechanisms of SSY on lipopolysaccharide (LPS)-induced acute lung injury (ALI) in rats. 48 rats were randomly divided into 4 groups: control (Ctrl) group, LPS-induced ALI group, low- (SSY-LD) and high- (SSY-HD) dose SSY-treated ALI group. SSY was administered to SSY-treated rats immediately after LPS induction. After 24 hours, blood gas analysis and lactate determination were performed; and bronchoalveolar lavage fluid was collected for detecting protein concentration and levels of cytokines. Lung tissues were obtained for Western blot analysis, histopathological analysis, wet-to-dry weight ratio calculation and measurement of oxidative stress levels. SSY improved oxygenation index and mean arterial pressure, decreased levels of lactate and heart rate, alleviated lung histopathology indexes including lung injury score, wet-to-dry weight ratio and exudation of protein as well as inflammatory cells in ALI rats. Furthermore, SSY reduced levels of pro-inflammatory and oxidative mediums, while increasing levels of anti-inflammatory cytokine and anti-oxidative activity in lung tissues. SSY also suppressed NF-KB signalling pathway and further activated Keap1-Nrf2-ARE signalling pathway activated by LPS. Moreover, all the effects caused by SSY in the SSY-HD group were more encouraging than those in the SSY-LD group. The results indicate that the preventive use of SSY can alleviate ALI through the anti-inflammatory and antioxidant effects mediated by inhibition of NF-KB signalling pathway and activation of Keap1-Nrf2-ARE signalling pathway, and the effect of high dose is better.

\section{Introduction}

Acute lung injury (ALI) is a life-threatening disease caused by a variety of intrapulmonary and extrapulmonary factors, leading to progressive hypoxemia, lung oedema and acute respiratory distress syndrome (ARDS), accounting for approximately $10 \%$ of ICU admissions worldwide [1]. Evidence indicates that inflammation and oxidative stress are the critical mechanisms behind it, resulting in diffuse alveolar exudation, hyaline membrane formation and alveolar and capillary epithelial damage and gradually initiating a pathophysiological process of ALI, including a ventilation/perfusion defect, worsened compliance and reduction of lung volume [2].

Current therapies for ALI mainly focus on anti-infection using antipathogenic microorganism drugs, antiinflammation with glucocorticoids and respiratory support with oxygen therapy and/or mechanical ventilation and have disadvantages including development of opportunistic infection, immunodeficiency, gastrointestinal hemorrhage and ventilator associated lung injury, which are vital reasons for high mortality of ALI/ARDS [1, 3]. Traditional Chinese medicine (TCM) preparations such as Jie-Geng-Tang, Shufeng Jiedu Capsule and Dachengqi decoction have emerged as promising therapies for ALI in animal models due to their high efficiency, low-cost, easy accessibility and low adverse effect rate [4-6]. Shen-suyin (SSY, Jinsoin in Japanese, Samsoeum in Korean) is a traditional Chinese herb formula consisting of 13 kinds of Chinese herbs, which was first recorded in the Prescriptions of the Bureau of Taiping People's Welfare Pharmacy in Song Dynasty in China. It is mainly used in the treatment of respiratory 
inflammatory diseases, including common cold, pneumonia, laryngitis and pharyngitis, and has a wide range of pharmacological activities, including anti-allergic, anti-inflammatory, anti-asthmatic and immunomodulatory effectse [7-9].

Considering these properties, we hypothesised that SSY might provide beneficial effects for ALI. However, as far as we know, there are no current studies reporting the therapeutic effects of SSY on ALI, nor yet its possible molecular mechanisms. Therefore, this study was conducted to investigate its potential effects and mechanisms on rats with lipopolysaccharide (LPS)-induced ALI.

\section{Materials And Methods}

\section{Preparation of SSY}

All the herbs purchased from Beijing Tong Ren Tang Co., Ltd. (Beijing, China) were prepared according to the original SSY prescription (Table 1) in the Prescriptions of the Bureau of Taiping People's Welfare Pharmacy. The crude herbs were chopped, mixed and soaked with $750 \mathrm{ml}$ distilled water at room temperature for $30 \mathrm{~min}$. Then, they were boiled at $100^{\circ} \mathrm{C}$ for another $30 \mathrm{~min}$ in an extractor to obtain SSY decoction. We collected, filtered and evaporated the decoction to $100 \mathrm{ml}(2.5 \mathrm{~g}$ crude herbs was extracted to $1 \mathrm{ml}$ decoction) to further investigation.

\section{Animals}

7-week-old male Sprague-Dawley (SD) rats (200-230 g) were purchased from the Shanghai SLACCAS Laboratory Animal Co., Ltd. (Shanghai, China). They had free access to chow and water and were housed in a standard animal facility (temperature, between $22^{\circ} \mathrm{C}$ and $24^{\circ} \mathrm{C}$; humidity, $50 \%-60 \%$; day-night cycle, 12:12). After 7-day acclimatisation, we randomly divided 48 rats into 4 groups ( $n=12 /$ group): control (Ctrl group), LPS-induced ALI (ALI group), low-dose (SSY-LD group) and high-dose (SSY-HD group) SSYtreated ALI group. We injected rats in ALI and SSY-treated groups with a single dose of $7.5 \mathrm{mg} / \mathrm{kg}$ LPS (Sigma-Aldrich Co, St. Louis, MO, USA) through the tail vein to induce ALI as described [10] and injected rats in Ctrl group with an equivalent volume of normal saline (NS). Immediately after LPS administration, we administered SSY-LD group rats an animal equivalent dose (AED) of SSY $(10.28 \mathrm{ml} / \mathrm{kg})+20.56 \mathrm{ml} / \mathrm{kg}$ NS and SSY-HD group rats 3 times of AED $(30.84 \mathrm{ml} / \mathrm{kg})$ by gavage, and rats in Ctrl and ALI groups were given $30.9 \mathrm{ml} / \mathrm{kg}$ NS. AED was calculated as follows: AED $(\mathrm{g} / \mathrm{kg})=$ Human dose $(\mathrm{g} / \mathrm{kg}) \times 6.17$ [11]. Human dose used in this study was converted into $4.17 \mathrm{~g} / \mathrm{kg}$ (Human dose = Total drug weight/Body weight $=250 \mathrm{~g} / 60 \mathrm{~kg}=4.17 \mathrm{~g} / \mathrm{kg}$ ) according to the Prescriptions of the Bureau of Taiping People's Welfare Pharmacy.

After $24 \mathrm{~h}$, we anaesthetised all rats with $3 \%$ pentobarbital sodium $(50 \mathrm{mg} / \mathrm{kg})$ and measured their mean arterial pressure (MAP) and heart rate (HR) from the carotid artery using a PowerLab data acquisition system (AD Instruments, Sydney, Australia). After collecting hemodynamic data, we collected the arterial blood for blood gas analysis and lactic acid determination. We calculated oxygenation index (OI) using 
the following equation: Ol=Arterial partial pressure of oxygen $\left(\mathrm{PaO}_{2}\right)$ /Fraction of inspired oxygen $\left(\mathrm{FiO}_{2}\right)$. Finally, we sacrificed all animals by exsanguination.

The animal protocol was in compliance with the Guide for the Care and Use of Laboratory Animals published by the US National Institute of Health (NIH Publication No. 85-23, revised 1996) and was approved by the Experimental Animal Care Committee of the Fujian Provincial Hospital (Fuzhou, China).

\section{Bronchoalveolar lavage fluid (BALF) tests}

We performed bronchoalveolar lavage (BAL) in the left lung of rats; repeated them twice using $2 \mathrm{ml} N S$ each time; mixed the retrieved lavage samples and centrifuged $\left(200 \times \mathrm{g}\right.$ for $10 \mathrm{~min}$ at $\left.4^{\circ} \mathrm{C}\right)$ them before further investigations. We detected protein concentration and cytokines levels (TNF- $a, I L-1 \beta, ~ I L-6, C X C L 2$ and IL-10) in the supernatant using a bicinchoninic acid (BCA) protein assay kit (Thermo Fisher Scientific, MA, USA) and enzyme-linked immunosorbent assay (ELISA) kits (Abcam, Cambridge, MA, USA), according to the manufacturer's introduction. We re-suspended the pellets in $1.0 \mathrm{ml}$ phosphate buffered solution (PBS) and used a cell counter to determine the total number of nucleated cells in BALF. The resuspended BALF was then centrifuged $\left(200 \times g\right.$ for $10 \mathrm{~min}$ at $\left.4^{\circ} \mathrm{C}\right)$ onto slides. We stained the slides with a Wright-Giemsa stain kit (Nanjing Jiancheng Bioengineering Institute, Nanjing, China) to further count the number of neutrophils using a haemocytometer.

\section{Histopathology and wet-to-dry (W/D) weight ratio analysis}

We excised and immediately weighed the middle lobe of the right lung to measure its wet weight and placed the lung sample in an oven at $65^{\circ} \mathrm{C}$ for $60 \mathrm{~h}$ for dry weight detection. For histopathological analysis, we harvested lung tissues and fixed them with $10 \%$ neutral formalin ( $\mathrm{pH} \mathrm{7.40),} \mathrm{embedded} \mathrm{them}$ into paraffin and sectioned them (4- $\mu \mathrm{m}$ slices). After deparaffinization, we stained the sections with haematoxylin and eosin (Nanjing Jiancheng Bioengineering Institute) for microscopic observation (Nikon, Tokyo, Japan). We semi-quantitatively scored the injury degree of each tissue slice using the following published criteria: 1 , no injury; 2 , injury $\leq 25 \%$ of the field; 3 , injury $\leq 50 \%$ of the field; 4 , injury $\leq 75 \%$ of the field and 5, diffuse injury [12]. Three pathologists blinded to the experimental procedures analysed each slice, and we calculated the average score as the final lung injury score.

\section{Oxidative stress and superoxide dismutase (SOD) activity measurements}

We homogenised lung tissues in PBS; centrifuged $\left(1000 \times \mathrm{g}\right.$ for $10 \mathrm{~min}$ at $\left.4^{\circ} \mathrm{C}\right)$ the samples; collected the supernatant for detecting peroxidation products using hydroxyl free radical $(\cdot \mathrm{OH}$; Nanjing Jiancheng Bioengineering Institute), hydrogen peroxide $\left(\mathrm{H}_{2} \mathrm{O}_{2} ; \mathrm{Abcam}\right)$ and malondialdehyde (MDA; Abcam) assay kits and evaluated antioxidant activity using a commercial SOD activity assay kit (Abcam), according to manufacturers' instructions.

\section{Western blot analysis}


We homogenised the stored frozen lung tissues in ice-cold lysis buffer and centrifuged $(12000 \times g$ for 10 min at $4^{\circ} \mathrm{C}$ ) the samples. After 10 -min boiling, we determined protein concentrations using a BCA protein assay kit (Thermo Fisher Scientific), separated the protein samples $(20 \mu \mathrm{g})$ in $10 \%$ SDS-PAGE and transferred them onto PVDF membranes. We blocked membranes with $5 \%$ fat-free milk at room temperature for $1 \mathrm{~h}$ and then incubated overnight at $4^{\circ} \mathrm{C}$ with their respective primary antibodies [mouse anti- $\beta$-actin (1:1000; Santa Cruz Biotechnology, Santa Cruz, CA, USA); rabbit anti-NF-KB p65/Phospho-NFKB p65/IKBa/Phospho-IKBa (1:1000/1:1000/1:1000/1:1000; Cell Signaling Technology, Beverly, MA, USA); rabbit anti-IKKß/Phospho-IKKß (1:1500/1:500; Abcam) or rabbit anti-Keap1/Nrf2/NQ01/GCLC/HO1 (1:500/1:1500/1:10000/1:400/1:2000; Abcam)]. After three washes, the blots were incubated with HRPlabelled goat anti-mouse/rabbit IgG secondary antibody (1:5000/1:2000; Abcam) for $1 \mathrm{~h}$ at room temperature. Then, we washed the membranes three times again and exposed them to a chemiluminescence luminol solution. Finally, we obtained the blots using a ChemiDoc XRS imaging system (Bio-Rad, California, American) and measured the band densities using an ImageJ software (National Institutes of Health, USA). $\beta$-actin was used as an internal reference.

\section{Statistical analysis}

We expressed all quantitative data as mean $\pm S D$. We compared variables among groups by analysis of variance (one-way ANOVA), followed by a least significant difference LSD-t-test for multiple comparisons. We analysed all statistical data using the SPSS 13.0 software (IBM, Armonk, NY, USA). P-values $<0.05$ were considered statistically significant.

\section{Results}

\section{Effects of SSY on vital signs and on blood lactate level}

All animals survived at 24h. LPS-injected rats presented a series of physical signs of illness, e.g. polypnea, bradykinesia and lethargy. SSY-LD and SSY-HD group rats showed similar signs but with less severity (especially SSY-HD group rats). Twenty-four hours after LPS induction, the OI and MAP in ALI rats significantly decreased, whereas the HR and blood lactate concentration (a marker for histanoxia and circulatory disturbances) increased compared with those of control rats. Compared with ALI group rats, SSY-LD group rats had increased OI and MAP and presented reduced HR and blood lactate levels. Moreover, the $\mathrm{OI}$ and MAP increased and the HR and lactate levels were lower in SSY-HD group than in SSY-LD group (Figure. 1A-D). Overall, these results showed that SSY could relieve the respiratory and circulatory dysfunction in LPS-induced ALI rats.

\section{SSY improved lung histopathological changes in ALI rats}

After $24 \mathrm{~h}$, the lung tissues from rats with untreated ALI showed severe epithelial cell necrosis, alveolar exudation, haemorrhage and inflammatory cell infiltration, with capillary congestion, interstitial oedema, thickened alveoli septa and small airway occlusion. Conversely, although SSY-treated group rats also displayed slight-to-moderate lung injury, the severity (especially in SSY-HD group) was significantly less 
than that in untreated ALI group rats (Figure. 1E). We further quantified the effect of SSY on LPS-induced lung injury by comparing values for the lung W/D ratio (an index for tissue oedema), total protein in BALF (an epithelial permeability marker) and lung injury score. LPS caused significantly higher levels of W/D ratio, protein exudation and lung injury score in ALI rats than in control rats. All of these indicators decreased, especially at a high dose, after SSY treatment, indicating that SSY alleviated the lung tissue pathology in ALI rats (Figure. 1F-H).

\section{SSY attenuated lung inflammation associated with LPS}

LPS caused lung infiltration of total leucocytes and neutrophils and increased expression of proinflammatory mediators including CXCL2 (MIP-2 in mouse), TNF-a, IL-1 $\beta$ and IL- 6 in the BALF after $24 \mathrm{~h}$. SSY partially prevented rats in ALI group from inflammatory reaction, and the effect was observed more obvious in SSY-HD group than in SSY-LD group (Figure. 2A-F). LPS also resulted in increased IL-10 levels, an anti-inflammatory cytokine, and SSY intervention further increased the IL-10 level. Moreover, the IL-10 level in the SSY-HD group was higher than that in the SSY-LD group (Figure. 2G).

\section{SSY alleviated oxidative stress levels in rats with ALI}

Levels of $\mathrm{H}_{2} \mathrm{O}_{2}, \cdot \mathrm{OH}$ and MDA (oxidative stress indicators) had significantly increased in lung tissues of ALI group rats than in those of control rats, but the alterations were less obvious in rats treated with SSY (Figure. 2H-J). SOD activity (a biomarker for anti-oxidation) increased in ALI group rats compared with that in control group rats, and, interestingly, SSY seemed to further enhance its activity (Figure. 2K). In addition, level of oxidative stress was lower and antioxidant activity higher in SSY-HD group rats than in SSY-LD group rats (Figure. 2H-K).

\section{Anti-inflammatory effect of SSY can be attributed to NF-KB signalling pathway suppression}

The NF-KB signalling pathway is pivotal during inflammation [13]. After 24-h LPS induction, the phosphorylation levels of IKKB, IkBa and p65 (a subunit of NF-KB) in ALI group rats were remarkably elevated. Meanwhile, the total IkBa level had notably reduced in ALI rats compared with control rats, suggesting enhanced degradation. However, intervention with two SSY doses significantly inhibited these alterations, and a more significant effect was observed in the SSY-HD group than in the SSY-LD group (Figure. 3A-E).

\section{Nrf2 signalling pathway activation by SSY mediates its antioxidant effects}

The Keap1-Nrf2-ARE signalling pathway is a vital molecular mechanism associated with antioxidant stress [14]. LPS downregulated Keap1 expression in lung tissues and upregulated expression of Nrf2, NQ01, GCLC and HO-1 in LPS-treated rats. Remarkably, SSY treatment further weakened Keap1 expression and promoted expression of Nrf2, NQ01, GCLC and HO-1. Moreover, expression of Keap1 was significantly lower and levels of Nrf2, NQ01, GCLC and HO-1 were higher in SSY-HD group than in SSY-LD group (Figure. 4A-F). 


\section{Discussion}

Our experiments showed that LPS treatment resulted in severe microstructure damage, oedema and elevated lung tissue permeability, leading to respiratory and circulatory disorders as previously reported $[10,12,15]$. We demonstrated for the first time that prophylactic treatment using SSY prevented respiratory and circulatory functions from damage by LPS through alleviating pathological changes of the lungs in ALI rats. The underlying mechanisms possibly involved anti-inflammation via inhibition of NF-KB signalling pathway and antioxidant stress via activation of the Keap1-Nrf2-ARE signalling pathway, which seemed more effective at a high dose.

ALI triggers excessive inflammation and uncontrolled oxidative stress, producing several proinflammatory cytokines and free radicals, but also promotes anti-inflammatory and anti-oxidative responses, possibly acting as compensatory mechanisms to defend against these harmful reactions [10, $16,17]$. Consistent with other studies [16, 17], we found that LPS exposure resulted in high-level inflammatory infiltration and oxidative stress and led to elevated levels of anti-inflammatory and antioxidative factors. However, the compensating mechanisms are often insufficient and ALI exacerbations are common. If inadequately treated, it causes gradual dysfunction of extrapulmonary organs (such as heart, liver and brain) and subsequently causes multiple organ failure and death $[18,19]$.

Most Chinese herbs present in SSY, including Panax ginseng [20], Folium perillae [21], Pueraria lobata [22], Poria cocos [23], Fructus aurantii [24], Platycodon grandiflorum [25], pericarpium citri reticulatae [26], Glycyrrhiza uralensis fisch [27], Costus root [28], Zingiber Officinale Radix [29] and Zizyphi fructus [30], have shown anti-inflammatory and/or anti-oxidative effects. Additionally, Peucedani radix, another component of SSY, exhibits a wide spectrum of pharmacological activities including anti-infective, antitussive, anti-asthmatic and expectorant effects, which help relieve symptoms and improve ALI prognosis [31]. In addition, some components of SSY alleviate multiple organ dysfunction triggered by ALI. For example, Pinellia ternate, possessing sedative, hypnotic and anticonvulsant effects, can relieve neuropsychiatric symptoms [32], and Panax ginseng, as well as Glycyrrhiza uralensis fisch, enjoys popularity for their hepatoprotective effects [20,33]. Based on these pharmacological characteristics, it is reasonable to speculate that the mechanisms resulting in ALI attenuation by SSY involve antiinflammation, anti-oxidation and organ protection, as our experimental results suggest. To compare different doses of SSY treatment, we used low and high SSY doses for intervention after LPS injection. Both doses could restore lung tissue impairment, but high dose had a more pronounced curative effect, suggesting a potential dose-response relationship between SSY and its therapeutic effects. The characteristic early lesions in ALI include mostly tissue injury (rather than necrocytosis) and mild-tomoderate (reversible) organ function damage $[34,35]$. If left to progress, ALI results in tissue necrocytosis, causing serious and irreversible organ dysfunctions that are difficult to reverse medically [34,35]; thus, we preventively treated rats in the early stage of the ALI instead of those with middle or advanced stages of the disease. 
The inactive form of NF-KB, a nuclear transcription factor, is located in the cytoplasm and bound by a variety of inhibitory proteins, the NF-KB inhibitors (IkBs) family. Upon exposure to various stimuli, the IkB kinase complex (IKK) is activated into phospho-IKK, which further phosphorylates IkBs and leads to its ubiquitination and degradation. Without inhibition of $\mathrm{kBBs}, \mathrm{NF}-\mathrm{kB}$ is phosphorylated and translocates to the nucleus, promoting transcription of inflammation-related genes [13]. Our results are consistent with those of studies showing that LPS stimulates phosphorylation of IKK $\beta$, IkBa and p65 and promotes expression of pro-inflammatory factors, indicating NF-KB signalling pathway activation $[10,12,15]$. Our results suggest that SSY suppresses this signalling pathway markedly, especially at a high dose. Among the medicinal herbal concoctions based on SSY, some have been reported to promote IL-10 expression $[24,36]$. For example, the mechanism that Fructus aurantii exhibited anti-inflammatory effects in LPStreated mice was partially through IL-10 expression stimulation [24]. Overall, the LPS-induced high level of IL-10 $[10,16,17,24]$, which was further increased by SSY treatment in our study, suggests an amplification of the anti-inflammatory reaction, but the molecular mechanism involved remains to be elucidated.

Numerous antioxidative metabolites such as NQ01, GCLC and HO-1 are produced as a result of the body's antioxidant system being activated by ALI to protect against oxidative stress damage. Nrf2, which is kept in the cytoplasm by Keap1 under unstressed conditions, is a transcription factor that regulates expression of antioxidant proteins such as NQ01, GCLC and HO-1 by travelling to nucleus where it binds to antioxidant response elements (AREs) and initiates transcription of antioxidant genes [14]. Our results agree with those of published studies showing that LPS leads to increased production of oxidative metabolites, activating the Keap1-Nrf2-ARE pathway and increasing antioxidant activity $[14,16]$. An innovative finding is that prophylactic treatment by SSY further increases antioxidant activity in LPStreated rats and reduces oxidative stress levels; our evidence suggests this occurs by SSY's activation of the Nrf2 signalling pathway in a possible dose-dependent manner.

Our results were in accordance with previous studies that inhibition of both inflammation and oxidative stress could attenuate LPS-induced ALI effectively [37, 38]. The molecular mechanisms were involved in NF-KB and Nrf2 signaling pathway, respectively [37, 38]. Besides, their synergistic effect in attenuation of ALI has also been reported $[39,40]$. Our study indicates SSY may be worthy of further investigation into a therapeutic agent for ALI.

The major limitation of this study is that rodent model can not fully mimic human disease. Although the results of current study are encouraging, the clinical significance remains to be further investigated. SSY has been used in China for thousands of years. Nevertheless, many questions about its effects are still unclear, especially regarding pharmacological properties. Further research is needed to elucidate pharmacological activities and side effects of the components in the herbal formula.

In summary, preventive use of low- and high-dose SSY treatment stabilised vital signs and attenuated lung histopathology in LPS-induced ALI in rats, and high dose tended to be more effective. The main 
acting mechanisms included anti-inflammation and antioxidant stress mediated by inhibition of NF-KB signalling pathway and activation of Nrf2 signalling pathway, respectively.

\section{Declarations}

\section{Acknowledgments}

This study was sponsored by the Startup Fund for scientific research, Fujian Medical University (Grant number: 2018QH1123).

\section{References}

1. Fan E, Brodie D and Slutsky AS. Acute Respiratory Distress Syndrome: Advances in Diagnosis and Treatment. JAMA 2018;319:698-710.

2. Dias-Freitas F, Metelo-Coimbra $C$ and Roncon-Albuquerque $R$ Jr. Molecular mechanisms underlying hyperoxia acute lung injury. Respir Med 2016;119:23-28.

3. Lewis SR, Pritchard MW, Thomas CM and Smith AF. Pharmacological agents for adults with acute respiratory distress syndrome. Cochrane Database Syst Rev 2019;7:CD004477.

4. Yuan Y, Liao Q, Xue M, Shi Y, Rong L, Song Z, Tong Z, Zheng W, Zhu Q, Cui X and Tao Z. Shufeng Jiedu Capsules Alleviate Lipopolysaccharide-Induced Acute Lung Inflammatory Injury via Activation of GPR18 by Verbenalin. Cell Physiol Biochem 2018;50:629-639.

5. Liu Y, Hong Z, Qian J, Wang Y and Wang S. Protective effect of Jie-Geng-Tang against Staphylococcus aureus induced acute lung injury in mice and discovery of its effective constituents. J Ethnopharmacol 2019;243:112076.

6. Liu G, Liu F, Xiao L, Kuang Q and Yu Y. Treatment of hyperlipidemic acute pancreatitis with modified Dachengqi decoction combining with conventional therapy based on "six-hollow-organs to be unblocked" theory. Annals of palliative medicine, 2020;9(4):2045-2053.

7. Jeon WY, Shin IS, Shin HK and Lee MY. Samsoeum water extract attenuates allergic airway inflammation via modulation of Th1/Th2 cytokines and decrease of iNOS expression in asthmatic mice. BMC Complement Altern Med 2015;15:47.

8. Jeong SJ, Yoo SR, Seo CS and Shin HK. Traditional Korean Herbal Formula Samsoeum Attenuates Adipogenesis by Regulating the Phosphorylation of ERK1/2 in 3T3-L1 Cells. Evid Based Complement Alternat Med 2015;2015:893934.

9. Kim A, Yim NH and Ma JY. Samsoeum, a traditional herbal medicine, elicits apoptotic and autophagic cell death by inhibiting Akt/mTOR and activating the JNK pathway in cancer cells. BMC Complement Altern Med 2013;13:233.

10. Li Y, Zeng Z, Cao Y, Liu Y, Ping F, Liang M, Xue Y, Xi C, Zhou M and Jiang W. Angiotensin-converting enzyme 2 prevents lipopolysaccharide-induced rat acute lung injury via suppressing the ERK1/2 and NF-kB signaling pathways. Sci Rep 2016;6:27911. 
11. Shin JW, Seol IC. Interpretation of animal dose and human equivalent dose for drug development. J Korean Orient Med. 2010;31:1-7.

12. Tong L, Bi J, Zhu X, Wang G, Liu J, Rong L, Wang Q, Xu N, Zhong M, Zhu D, SongY and Bai C. Keratinocyte growth factor-2 is protective in lipopolysaccharide-induced acute lung injury in rats. Respir Physiol Neurobiol 2014;201:7-14.

13. Fiordelisi A, laccarino G, Morisco C, Coscioni E and Sorriento D. NFkappaB is a Key Player in the Crosstalk between Inflammation and Cardiovascular Diseases. Int J Mol Sci 2019;20:1599.

14. Bellezza I, Giambanco I, Minelli A and Donato R. Nrf2-Keap1 signaling in oxidative and reductive stress. Biochim Biophys Acta Mol Cell Res 2018;1865:721-733.

15. Zheng Y, Liu S, Fan C, Zeng H and Yu L. Holistic quality evaluation of Qingwen Baidu Decoction and its anti-inflammatory effects. Journal of Ethnopharmacology, 2020, 263:113145.

16. Li J, Li D, Liu X, Tang S and Wei F. Human umbilical cord mesenchymal stem cells reduce systemic inflammation and attenuate LPS-induced acute lung injury in rats. J Inflamm (Lond) 2012;9:33.

17. Zhou H, Chen M, Zhang G and Ye RD. Suppression of Lipopolysaccharide-Induced Inflammatory Response by Fragments from Serum Amyloid A. J Immunol 2017;199:1105-1112.

18. Bomsztyk K, Mar D, An D, Sharifian R, Mikula M, Gharib SA, Altemeier WA, Liles WC and Denisenko 0. Experimental acute lung injury induces multi-organ epigenetic modifications in key angiogenic genes implicated in sepsis-associated endothelial dysfunction. Crit Care 2015;19:225.

19. Mai N, Prifti L, Rininger A, Bazarian $\mathrm{H}$ and Halterman MW. Endotoxemia induces lung-brain coupling and multi-organ injury following cerebral ischemia-reperfusion. Exp Neurol 2017;297:82-91.

20. Lam P, Cheung F, Tan HY, Wang N, Yuen MF and Feng Y. Hepatoprotective Effects of Chinese Medicinal Herbs: A Focus on Anti-Inflammatory and Anti-Oxidative Activities. Int J Mol Sci 2016;17:465.

21. Kong X, Huo G, Liu S, Li F, Chen W and Jiang D. Luteolin suppresses inflammation through inhibiting cAMP-phosphodiesterases activity and expression of adhesion molecules in microvascular endothelial cells. Inflammopharmacology 2019;27:773-780.

22. Liu Q, Wang C, Meng Q, Wu J, Sun H, Sun P, Ma X, Huo X and Liu K. Puerarin sensitized K562/ADR cells by inhibiting NF-KB pathway and inducing autophagy. Phytother Res. 2021 Mar;35(3):16581668.

23. Wu K, Fan J, Huang X, Wu X and Guo C. Hepatoprotective effects exerted by Poria Cocos polysaccharides against acetaminophen-induced liver injury in mice. Int J Biol Macromol 2018;114:137-142.

24. Li L, Chen J, Lin L, Pan G, Zhang S, Chen H, Zhang M, Xuan Y, Wang Y and You Z. Quzhou Fructus Aurantii Extract suppresses inflammation via regulation of MAPK, NF-KB, and AMPK signaling pathway. Sci Rep. 2020 Jan 31;10(1):1593.

25. Ke W, Wang P, Wang X, Zhou X, Hu X and Chen F. Dietary Platycodon grandiflorus Attenuates Hepatic Insulin Resistance and Oxidative Stress in High-Fat-Diet Induced Non-Alcoholic Fatty Liver Disease. Nutrients 2020;12:480. 
26. Yu X, Sun S, Guo Y, Liu Y, Yang D, Li G and Lü S. Citri Reticulatae Pericarpium(Chenpi): Botany, ethnopharmacology, phytochemistry, and pharmacology of a frequently used traditional Chinese medicine. J Ethnopharmacol 2018;220:265-282.

27. Yang R, Yuan BC, Ma YS, Zhou S and Liu Y. The anti-inflammatory activity of licorice, a widely used Chinese herb. Pharm Biol. 2017 Dec;55(1):5-18.

28. Choi DH, Kim JY, An JH, Sung SH and Kong HS. Effects of Saussurea costus on apoptosis imbalance and inflammation in benign prostatic hyperplasia. J Ethnopharmacol. 2021 Oct 28;279:114349.

29. Morvaridzadeh M, Sadeghi E, Agah S, Fazelian S, Rahimlou M, Kern FG, Heshmati S, Omidi A, Persad E and Heshmati J. Effect of ginger (Zingiber officinale) supplementation on oxidative stress parameters: A systematic review and meta-analysis. J Food Biochem. 2021 Feb;45(2):e13612.

30. Kim KI, Shin S, Lee N, Lee BJ, Lee J and Lee H. A traditional herbal medication, Maekmoondong-tang, for cough: A systematic review and meta-analysis. J Ethnopharmacol 2016;178:144-154.

31. Song Y, Jing W, Yan R and Wang Y. Research progress of the studies on the roots of Peucedanum praeruptorum dunn (Peucedani radix). Pak J Pharm Sci 2015;28:71-81.

32. Wu XY, Zhao JL, Zhang M, Li F, Zhao T and Yang LQ. Sedative, hypnotic and anticonvulsant activities of the ethanol fraction from Rhizoma Pinelliae Praeparatum. J Ethnopharmacol 2011;135:325-329.

33. Lu HF, Lai YH, Huang HC, Lee IJ, Lin LC, Liu HK, Tien HH and Huang C. Ginseng-plus-Bai-Hu-Tang ameliorates diet-induced obesity, hepatic steatosis, and insulin resistance in mice. J Ginseng Res 2020;44:238-246.

34. Lin L, Zhang L, Yu L, Han L, Ji W, Shen H and Hu Z. Time-dependent changes of autophagy and apoptosis in lipopolysaccharide-induced rat acute lung injury. Iran J Basic Med Sci 2016;19:632637.

35. Patel BV, Wilson MR, O'Dea KP and Takata M. TNF-induced death signaling triggers alveolar epithelial dysfunction in acute lung injury. J Immunol 2013;190:4274-4282.

36. Sun Y, Chen S, Wei R, Xie X, Wang C, Fan S, Zhang X, Su J, Liu J, Jia W and Wang X. Metabolome and gut microbiota variation with long-term intake of Panax ginseng extracts on rats. Food Funct 2018;9:3547-3556.

37. He YQ, Zhou CC, Yu LY, Wang L, Deng JL, Tao YL, Zhang F and Chen WS. Natural product derived phytochemicals in managing acute lung injury by multiple mechanisms. Pharmacol Res. 2021 Jan;163:105224.

38. Lei J, Wei Y, Song P, Li Y, Zhang T, Feng Q and Xu G. Cordycepin inhibits LPS-induced acute lung injury by inhibiting inflammation and oxidative stress. Eur J Pharmacol. 2018;818:110-114.

39. Köhler UA, Böhm F, Rolfs F, Egger M, Hornemann T, Pasparakis M, Weber A and Werner S. NF-KB/RelA and Nrf2 cooperate to maintain hepatocyte integrity and to prevent development of hepatocellular adenoma. J Hepatol. 2016;64:94-102.

40. Ma Z, Lu Y, Yang F, Li S, He X, Gao Y, Zhang G, Ren E, Wang Y and Kang X. Rosmarinic acid exerts a neuroprotective effect on spinal cord injury by suppressing oxidative stress and inflammation via 
modulating the Nrf2/HO-1 and TLR4/NF-KB pathways. Toxicol Appl Pharmacol. 2020;397:115014.

\section{Tables}

Table 1. The prescription of Shen-su-yin

\begin{tabular}{|c|c|c|c|}
\hline Herbal medicine & Chinese Pinyin & weight(g) & proportion $₫ \% \bigotimes$ \\
\hline Panax ginseng & Ren Shen & 23.4375 & 9.3750 \\
\hline Folium perillae & Zi Su Ye & 23.4375 & 9.3750 \\
\hline Pueraria lobata & Ge Gen & 23.4375 & 9.3750 \\
\hline Peucedani radix & Qian Hu & 23.4375 & 9.3750 \\
\hline Poria cocos & Fu Ling & 23.4375 & 9.3750 \\
\hline Pinellia ternate & Ban Xia & 23.4375 & 9.3750 \\
\hline Zingiber Officinale Radix & Sheng Jiang & 15.6250 & 6.2500 \\
\hline Zizyphi fructus & Da Zao & 15.6250 & 6.2500 \\
\hline Fructus aurantii & Zhi Qiao & 15.6250 & 6.2500 \\
\hline Platycodon grandiflorum & Jie Geng & 15.6250 & 6.2500 \\
\hline Pericarpium citri reticulatae & Chen Pi & 15.6250 & 6.2500 \\
\hline Glycyrrhiza uralensis fisch & Gan Cao & 15.6250 & 6.2500 \\
\hline Costus root & Mu Xiang & 15.6250 & 6.2500 \\
\hline Total & & 250.0000 & 100.00 \\
\hline
\end{tabular}

Figures 


\section{A}

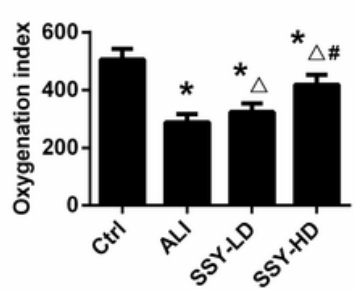

E

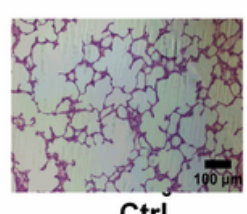

Ctrl

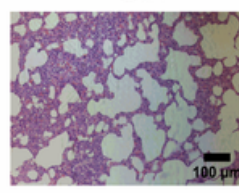

SSY-LD
B

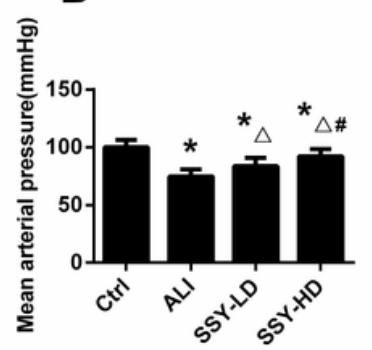

$\mathbf{F}$

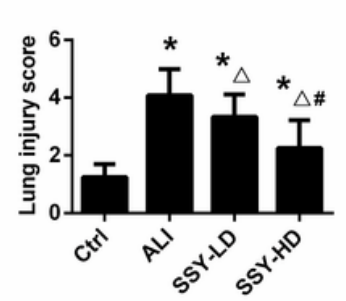

C

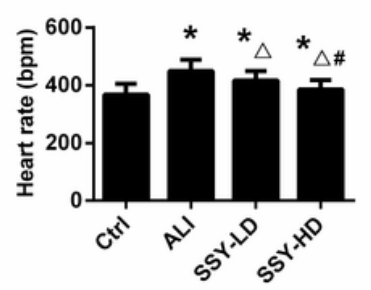

G

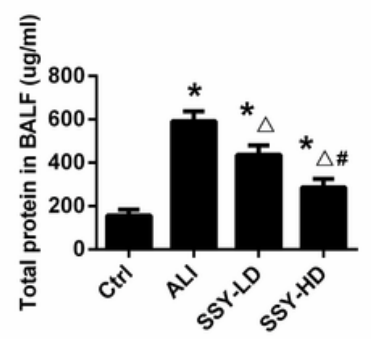

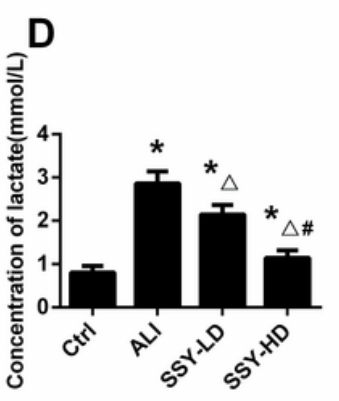

H

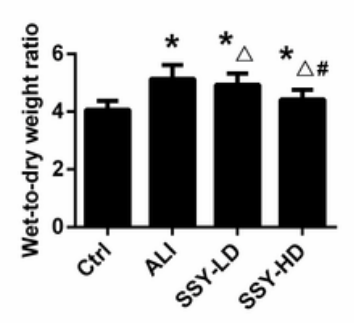

\section{Figure 1}

SSY improved respiratory and circulatory disorders in ALI rats (A-D) SSY improved the oxygenation index and mean arterial pressure in $A L I$ rats, and reduced the heart rate and level of lactate. $(E, F) 24$ hours after LPS administration, the HE $(\times 100)$ staining of lung sections in SSY-treated rats was less injured than that in ALI rats. Scale bar: $100 \mu \mathrm{m} .(\mathrm{G}, \mathrm{H})$ The BALF protein content and the wet-to-dry weight ratio were significantly reduced in the ALI rats given SSY than in those receiving only normal saline. Data are presented as mean $\pm S D(n=12)$. ${ }^{*} P<0.05$ vs. Ctrl group, $\triangle P<0.05$ vs. ALI group, $\# P<0.05$ vs. SSY-LD group. BALF, bronchoalveolar lavage fluid; SSY, Shen-su-yin; ALI, acute lung injury; LD, low-dose; HD, highdose. 


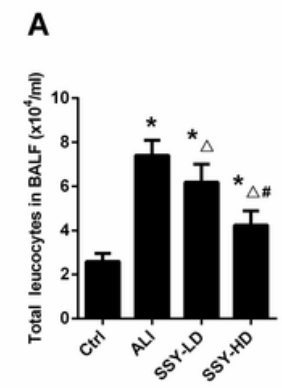

G

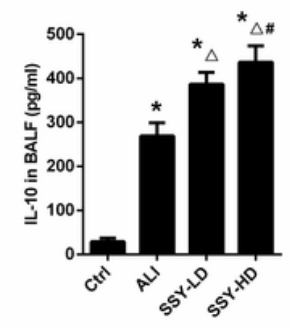

B

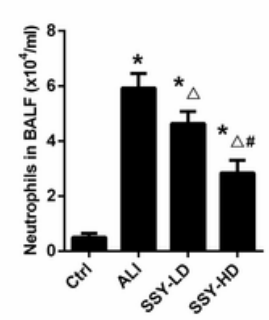

H

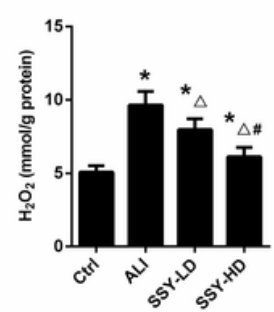

C

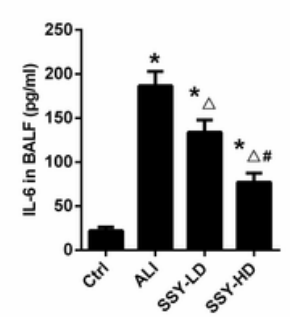

I

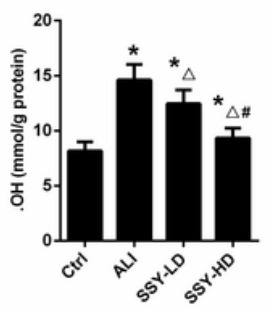

D

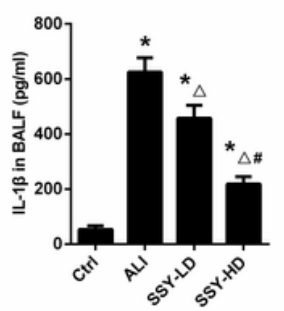

J

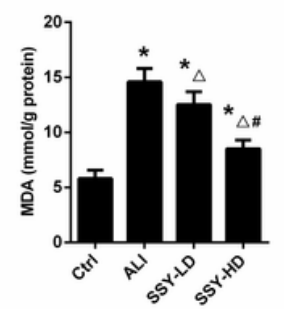

E

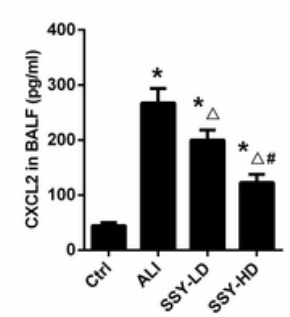

K

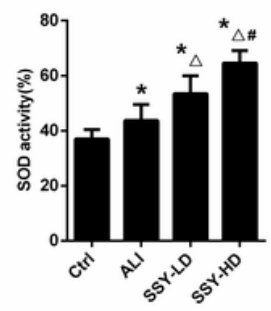

F

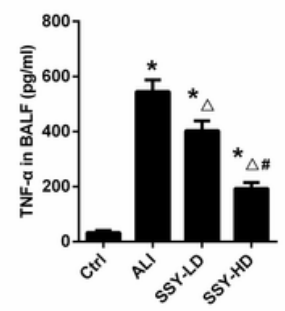

\section{Figure 2}

SSY alleviated inflammation and oxidative stress in LPS-induced ALI rats (A-G) SSY reduced levels of proinflammatory mediators and promoted expression of anti-inflammatory cytokine in ALI rats. (H-J) SSY suppressed levels of oxidative stress in SSY-treated groups. (K) LPS increased the SOD activity, and the activity was further elevated in SSY-treated groups. Data are presented as mean $\pm S D(n=12)$. *P $<0.05$ vs. Ctrl group, $\triangle P<0.05$ vs. ALI group, \#P<0.05 vs. SSY-LD group. BALF, Bronchoalveolar lavage fluid; IL, Interleukin; CXCL2, Chemokine (C-X-C motif) ligand 2; TNF, Tumor necrosis factor; H2O2, hydrogen peroxide; $\cdot \mathrm{OH}$, hydroxyl free radical; MDA, Malondialdehyde; SOD, superoxide dismutase; SSY, Shen-su-yin; ALI, acute lung injury; LD, low-dose; HD, high-dose. 


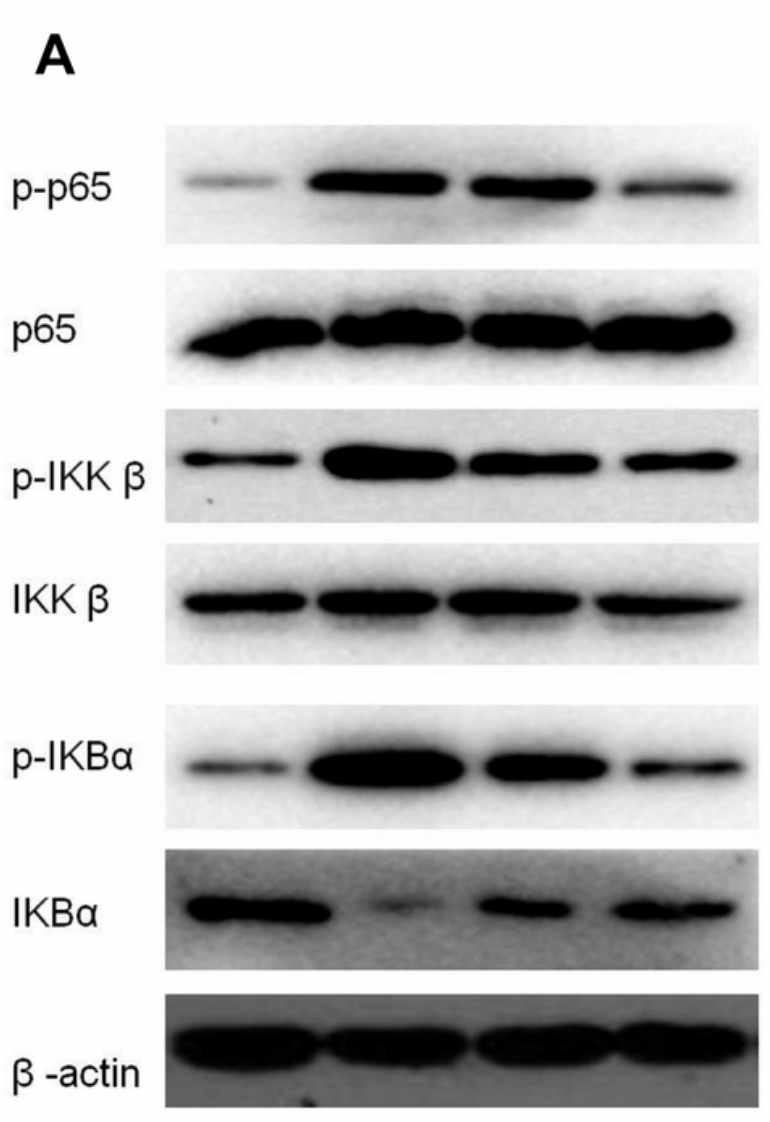

Ctrl
B

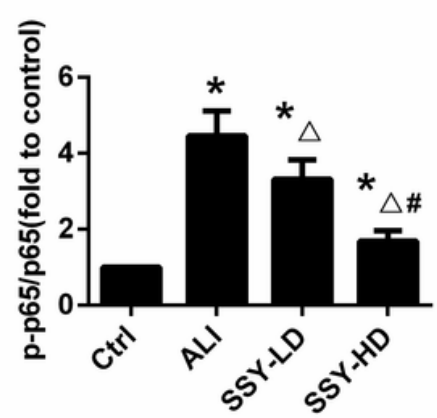

D

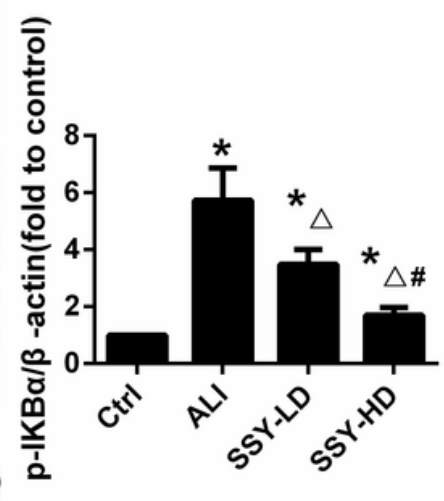

C

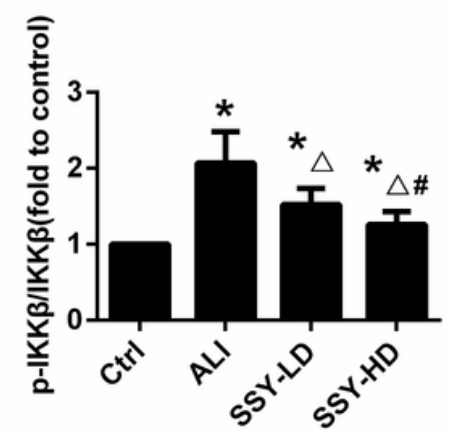

E

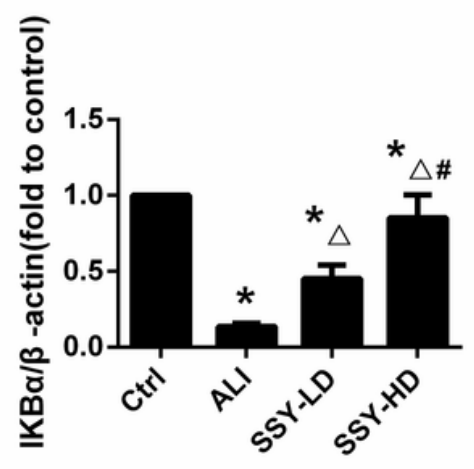

\section{Figure 3}

SSY suppressed NF-KB signalling pathway in rats with ALI (A) In vivo expression of $p-p 65, p 65, p-I K K \beta$, $I K K \beta, p-l k B a$ and $I k B a$ in lung tissues. (B-E) SSY decreased the phosphorylation of p65 (B), IKK $\beta$ (C) and $\mathrm{IKBa}(\mathrm{D})$ as well as the degradation of $\mathrm{IKBa}(\mathrm{E})$ in $\mathrm{ALI}$ rats. We used $\beta$-actin as an internal control. Data are presented as mean $\pm S D(n=8)$. ${ }^{*}<0.05$ vs. Ctrl group, $\triangle P<0.05$ vs. ALI group, $\# P<0.05$ vs. SSY-LD group. IkBa, NF-KB inhibitor $a$; IKK $\beta$, inhibitor of NF-KB kinase $\beta$; SSY, Shen-su-yin; ALI, acute lung injury; LD, low-dose; HD, high-dose. 
A

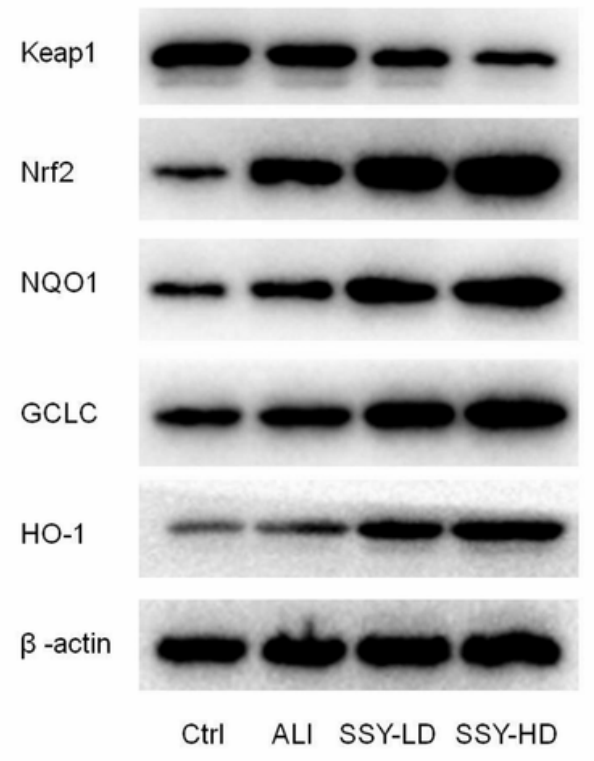

B
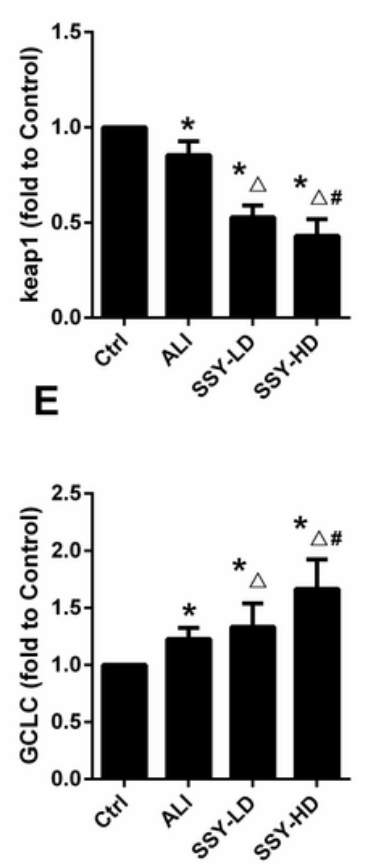

C
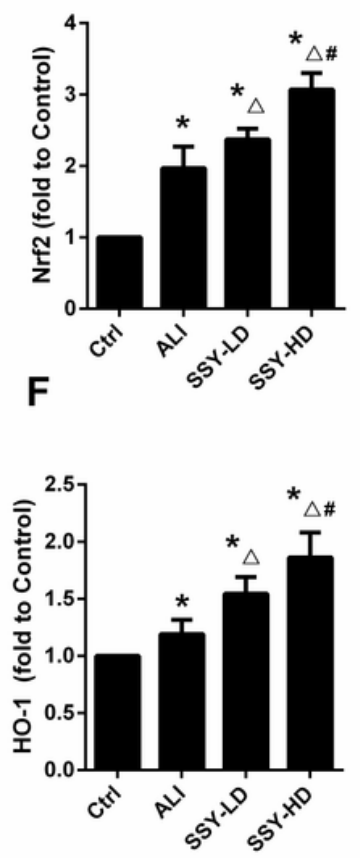

D

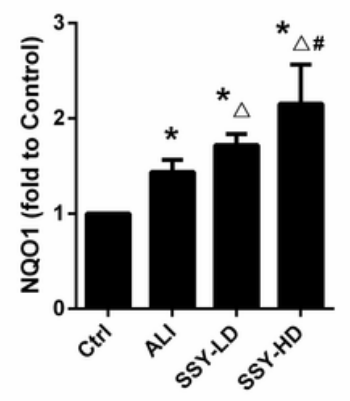

\section{Figure 4}

SSY activated Keap1-Nrf2-ARE signalling pathway in rats with ALI (A) Representative western blot analyses of Keap1, Nrf2, NQ01, GCLC and HO-1 in each group. (B-F) LPS administration downregulated the expression of Keap1 (B) and upregulated those of Nrf2 (C), NQ01 (D), GCLC (E) and HO-1 (F). The expression of Keap1 (B) was further decreased, while those of Nrf2 (C), NQ01 (D), GCLC (E) and HO-1 (F) were further increased after 24 hours of the SSY treatment. We used $\beta$-actin as an internal control. Data are presented as mean $\pm S D(n=8)$. ${ }^{*}<0.05$ vs. Ctrl group, $\triangle P<0.05$ vs. ALI group, $\# P<0.05$ vs. SSY-LD group. Keap1, Kelch-like ECH-associated protein 1; Nrf2, Nuclear factor (erythroid-derived 2)-like 2; NQ01, $\mathrm{NAD}(\mathrm{P}) \mathrm{H}$ :quinine oxidoreductase 1; GCLC, Glutamate-cysteine ligase catalytic subunit; $\mathrm{HO}-1$, Heme oxygenase 1; SSY, Shen-su-yin; ALI, acute lung injury; LD, low-dose; HD, high-dose. 